\title{
Neutrino oscillation processes in a quantum-field-theoretical approach
}

\author{
Vadim O. Egorov ${ }^{1,2}$ and Igor P. Volobuev ${ }^{1}$ \\ ${ }^{1}$ Skobeltsyn Institute of Nuclear Physics, Lomonosov Moscow State University 119991 Moscow, Russia \\ ${ }^{2}$ Faculty of Physics, Lomonosov Moscow State University, 119991 Moscow, Russia
}

(Received 3 October 2017; published 2 May 2018)

\begin{abstract}
It is shown that neutrino oscillation processes can be consistently described in the framework of quantum field theory using only the plane wave states of the particles. Namely, the oscillating electron survival probabilities in experiments with neutrino detection by charged-current and neutral-current interactions are calculated in the quantum field-theoretical approach to neutrino oscillations based on a modification of the Feynman propagator in the momentum representation. The approach is most similar to the standard Feynman diagram technique. It is found that the oscillating distance-dependent probabilities of detecting an electron in experiments with neutrino detection by charged-current and neutral-current interactions exactly coincide with the corresponding probabilities calculated in the standard approach.
\end{abstract}

DOI: 10.1103/PhysRevD.97.093002

\section{INTRODUCTION}

Neutrino oscillation is a well-known and experimentally confirmed phenomenon, which is usually understood as the transition from a neutrino flavor state to another neutrino flavor state depending on the distance traveled [1-3]. However, the situation with the theoretical explanation of this phenomenon is paradoxical: there is no generally accepted explanation of this phenomenon, which is both quantum and relativistic, in the framework of quantum field theory, which is a synthesis of quantum mechanics and the special theory of relativity. Nowadays, the generally accepted explanation of this phenomenon is the quantum mechanical description in terms of plane waves based on the notion of neutrino flavor states. This standard approach is not perfect: the neutrino flavor states are superpositions of the neutrino mass eigenstates, and for this reason the processes with the flavor states cannot be consistently described within quantum field theory. The problem is the violation of energy-momentum conservation in such processes, because in local quantum field theory, where the four-momentum is conserved in any interaction vertex, different mass-eigenstate components of the flavor states must have different momenta as well as different energies. This problem was repeatedly discussed in the literature (see, e.g., [4-9]).

A possible solution to the problem with the violation of energy-momentum conservation is to go off the mass shell.

Published by the American Physical Society under the terms of the Creative Commons Attribution 4.0 International license. Further distribution of this work must maintain attribution to the author(s) and the published article's title, journal citation, and DOI. Funded by SCOAP ${ }^{3}$.
It was first discussed in Ref. [4], where it was suggested that the produced neutrino mass eigenstates are virtual and their motion to the detection point should be described by the Feynman propagators. In this approach, neutrino oscillations occur as a result of interference of the amplitudes of processes due to all three intermediate virtual neutrino mass eigenstates. However, the calculations of the amplitudes in this approach are essentially different from the standard calculations in the framework of the Feynman diagram technique in the momentum representation. This is due to the standard S-matrix formalism of QFT, which is not appropriate for describing processes at finite distances and finite time intervals. To describe the localized particles or nuclei, which produce and detect neutrinos, one has to use wave packets, which impede the calculation.

Later, this approach was developed in [5], where an important theorem was proved that the virtual particles propagating at macroscopic distances are almost on the mass shell. A detailed review of the approach can be found in $[6,7]$.

An approach combining the features of the one discussed above and of the standard approach was put forward in [8]. A crucial point of the approach is that neutrino oscillations are due to interference of the amplitudes, corresponding to the processes mediated by the neutrino mass eigenstates. However, unlike the approach discussed above, the authors treat the neutrinos to be detected as real particles and also make use of wave packets in their reasoning to disentangle the produced neutrino mass eigenstates and to account for energy-momentum conservation.

In the present paper, we will show that neutrino oscillation can be consistently described in the framework of quantum field theory using only plane wave states of the particles. Namely, we will explicitly calculate the 
probabilities of the neutrino oscillation processes in experiments with neutrino detection by charged-current and neutral-current interactions within a modified perturbative S-matrix formalism, which enables one to calculate the amplitudes of the processes passing at finite distances and finite time intervals. This formalism was put forward in [10]. It is based on the Feynman diagram technique in the coordinate representation [11] supplemented by new modified rules of passing to the momentum representation, which will be discussed below in detail.

\section{OSCILLATIONS IN EXPERIMENTS WITH NEUTRINO DETECTION BY CHARGED- CURRENT INTERACTION}

In the framework of the minimal extension of the Standard Model (SM) by the right neutrino singlets, we consider the case, where the neutrinos are produced and detected in the charged-current interaction with nuclei. After the diagonalization of the terms sesquilinear in the neutrino fields, the charged-current interaction Lagrangian of leptons takes the form

$L_{c c}=-\frac{g}{2 \sqrt{2}}\left(\sum_{i, k=1}^{3} \bar{l}_{i} \gamma^{\mu}\left(1-\gamma^{5}\right) U_{i k} \nu_{k} W_{\mu}^{-}+\right.$H.c. $)$,

where $l_{i}$ denotes the field of the charged lepton of the $\mathrm{i}$-th generation, $\nu_{i}$ denotes the field of the neutrino mass eigenstate most strongly coupled to $l_{i}$ and $U_{i k}$ stands for the Pontecorvo-Maki-Nakagawa-Sakata (PMNS) matrix. Due to this structure of the interaction Lagrangian any process involving the production of a neutrino at one point and its detection at another point, when treated perturbatively, can be represented in the lowest order by the following diagram,

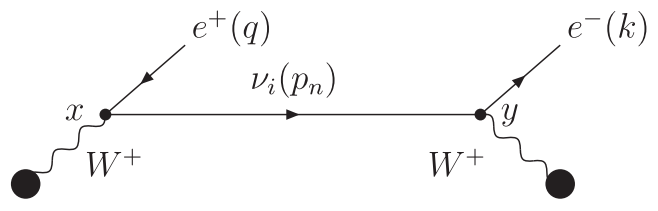

which should be summed over all three neutrino mass eigenstates. To be specific, we assume that the virtual $W$ bosons are produced and absorbed in interactions with nuclei. Namely, we suppose that a nucleus ${ }_{Z_{1}}^{A_{1}} X$ that will be called nucleus 1 radiates $W^{+}$boson and turns into the nucleus ${ }_{Z_{1}-1}^{A_{1}} X$ that will be called nucleus $1^{\prime}$, and a nucleus ${ }_{Z_{2}}^{A_{2}} X$ that will be called nucleus 2 absorbs $W^{+}$boson and turns into the nucleus ${ }_{Z_{2}+1}^{A_{2}} X$ that will be called nucleus $2^{\prime}$. Thus, the filled circles stand for the matrix elements of the charged weak hadron current

$$
j_{\mu}^{(1)}=\left\langle\left.{ }_{Z_{1}-1}^{A_{1}} X\left|j_{\mu}^{(h)}\right|\right|_{Z_{1}} ^{A_{1}} X\right\rangle, \quad j_{\rho}^{(2)}=\left\langle Z_{Z_{2}+1}^{A_{2}} X\left|j_{\rho}^{(h)}\right| X_{Z_{2}}^{A_{2}} X\right\rangle,
$$

associated with nuclei $1,1^{\prime}$ and $2,2^{\prime}$. As it is customary in QFT, we assume that the incoming nuclei 1 and 2 have definite momenta. Therefore all the three virtual neutrino eigenstates and the outgoing particles and nuclei also have definite momenta. In what follows, a 4-momentum of the virtual neutrino mass eigenstates defined only by the energy-momentum conservation in the production vertex will be denoted by $p_{n}$ and the one selected also by the experimental setting will be denoted by $p$.

The amplitude in the coordinate representation corresponding to diagram (2) can be written out in the standard way using the Feynman rules formulated in textbook [12]. According to the prescriptions of the standard perturbative S-matrix theory ([12], Sec. 24), in order to obtain the amplitude in the momentum representation, next we would have to integrate it with respect to $x$ and $y$ over the Minkowski space. However, in this case, we would get the amplitude of the process lasting an infinite amount of time and lose the information about the distance between the production and detection points defined by the experimental setting. In order to retain this information, we have to integrate with respect to $x$ and $y$ in such a way that the distance between these points along the direction of the neutrino propagation remains fixed. Of course, this is at variance with the standard S-matrix formalism. However, we recall that the diagram technique in the coordinate representation was developed by R. Feynman [11] without reference to S-matrix theory. Thus, the Feynman diagrams in the coordinate representation make sense beyond this theory, and for this reason we can integrate with respect to $x$ and $y$ in any way depending on the physical problem at hand. In particular, in the case under consideration, we have to integrate in such a way that the distance between the points $x$ and $y$ along the direction of the propagation of neutrino with momentum $\vec{p}$ is equal to the distance between a source and a detector, which will be denoted by $L$. This can be achieved by introducing the delta function $\delta(\vec{p}(\vec{y}-\vec{x}) /|\vec{p}|-L)$ into the integral. When passing to the momentum representation and integrating with respect to $x$ and $y$ over the Minkowski space, the introduction of this delta function is formally equivalent to replacing the standard Feynman propagator of the neutrino mass eigenstate $\nu_{i}$ in the coordinate representation $S_{i}^{c}(y-x)$ by $S_{i}^{c}(y-x) \delta(\vec{p}(\vec{y}-\vec{x}) /|\vec{p}|-L)$. However, we emphasize once again that the propagation of the neutrino mass eigenstates is still described by the Feynman propagator, and the introduced delta function serves only to represent the experimental situation that the neutrino mass eigenstates with momentum $\vec{p}$ are detected at a distance $L$ from the source.

The Fourier transform of the expression $S_{i}^{c}(y-x) \times$ $\delta(\vec{p}(\vec{y}-\vec{x}) /|\vec{p}|-L)$ was called in [10] the distancedependent propagator of the neutrino mass eigenstate $\nu_{i}$ in the momentum representation. It will be denoted by $S_{i}^{c}(p, L)$ and is defined by the integral: 


$$
S_{i}^{c}(p, L)=\int d x e^{i p x} S_{i}^{c}(x) \delta(\vec{p} \vec{x} /|\vec{p}|-L) .
$$

This integral can be evaluated exactly by the method of contour integration [10], and for $\vec{p}^{2}>m_{i}^{2}-p^{2}$ the result is given by

$$
\begin{aligned}
S_{i}^{c}(p, L)= & i \frac{\hat{p}+\vec{\gamma} \vec{p}\left(1-\sqrt{1+\frac{p^{2}-m_{i}^{2}}{\vec{p}^{2}}}\right)+m_{i}}{2 \sqrt{\vec{p}^{2}+p^{2}-m_{i}^{2}}} \\
& \times e^{-i\left(|\vec{p}|-\sqrt{\vec{p}^{2}+p^{2}-m_{i}^{2}}\right) L} .
\end{aligned}
$$

(In [10], this distance-dependent propagator was defined by substituting the dimensionless delta function $\delta(\vec{p} \vec{x}-|\vec{p}| L)$ into the integral, which results in an extra factor $|\vec{p}|$ in the denominator of $S_{i}^{c}(p, L)$. Below we will see that the present definition is more natural.) We emphasize that this distance-dependent fermion propagator makes sense only for macroscopic distances $L$.

The results of [5] imply that the virtual particles propagating at macroscopic distances are almost on the mass shell. This means that $\left|p^{2}-m_{i}^{2}\right| / \vec{p}^{2} \ll 1$ and we can expand the square roots to the first order in $\left(p^{2}-m_{i}^{2}\right) / \vec{p}^{2}$. It is clear that this term can be dropped everywhere, except in the exponential, where it is multiplied by a large macroscopic distance $L$. In this approximation distancedependent propagator (4) takes the simple form

$$
S_{i}^{c}(p, L)=i \frac{\hat{p}+m_{i}}{2|\vec{p}|} e^{-i \frac{m_{i}^{2}-p^{2}}{2|\vec{p}|} L} .
$$

It is worth noting that this distance-dependent fermion propagator taken on the mass shell has no pole and does not depend on the distance, which is also true for the exact propagator in formula (4).

In fact, we have discussed this distance-dependent propagator in order to explain better the motivations for introducing such an object, because it exactly corresponds to the experimental situation in neutrino oscillation processes. However, this distance-dependent propagator is not convenient for calculating amplitudes, because there is no inverse Fourier transformation for the propagator in formula (3). It turns out that a more convenient and a more fundamental object is the time-dependent propagator of the neutrino mass eigenstates, which can be defined as the Fourier transform of $S_{i}^{c}(x) \delta\left(x^{0}-T\right)$. A similar timedependent scalar field propagator was introduced in [10]. Using the results of the calculations of the time-dependent scalar field propagator in this paper, one can easily find that the time-dependent fermion field propagator in the momentum representation is

$$
\begin{aligned}
S_{i}^{c}\left(p_{n}, T\right)= & i \frac{\hat{p}_{n}-\gamma^{0}\left(p_{n}^{0}-\sqrt{\left(p_{n}^{0}\right)^{2}+m_{i}^{2}-p_{n}^{2}}\right)+m_{i}}{2 \sqrt{\left(p_{n}^{0}\right)^{2}+m_{i}^{2}-p_{n}^{2}}} \\
& \times e^{i\left(p_{n}^{0}-\sqrt{\left.\left(p_{n}^{0}\right)^{2}+m_{i}^{2}-p_{n}^{2}\right)} T\right.} .
\end{aligned}
$$

The advantage of the time-dependent propagator is that there exists the inverse Fourier transformation of this propagator, which allows one to retain the standard rules of the Feynman diagram technique just by replacing the Feynman propagator by this time-dependent propagator. For macroscopic time intervals $T$, i.e., for the particles close to the mass shell, it looks explicitly like

$$
S_{i}^{c}\left(p_{n}, T\right)=i \frac{\hat{p}_{n}+m_{i}}{2 p_{n}^{0}} e^{-i \frac{m_{i}^{2}-p_{n}^{2}}{2 p_{n}^{0}} T} .
$$

In case all the neutrinos in a beam have the same momentum $p$ defined by the experimental setting, we can express the time $T$ in terms of the distance $L$ and the neutrino speed $|\vec{p}| / p^{0}$ as $T=L p^{0} /|\vec{p}|$, neglect the neutrino mass that is small compared to $\hat{p}$ and get a distance-dependent propagator,

$$
S_{i}^{c}(p, L)=i \frac{\hat{p}}{2 p^{0}} e^{-i \frac{m_{i}^{2}-p^{2}}{2|\vec{p}|} L},
$$

which coincides with the above defined distance-dependent propagator of neutrinos (5) in the approximation of small neutrino masses. In what follows, we will use propagators (7), (8) for describing neutrino oscillation processes. We also note that the time-dependent scalar field propagator is adequate for calculating the probabilities of oscillation processes with massive scalar mesons, where we cannot neglect their masses.

Now we will calculate the amplitude in the momentum representation corresponding to diagram (2) in the case, where the time difference $y^{0}-x^{0}$ is fixed and equal to $T$. Since the momentum transfer in both production and detection processes is small, we can use the approximation of Fermi's interaction. Then making use of time-dependent propagator (7) and keeping the neutrino masses only in the exponential we can explicitly write out the amplitude in the momentum representation corresponding to diagram (2) summed over all three neutrino mass eigenstates:

$$
\begin{aligned}
M= & -i \frac{G_{F}^{2}}{4 p_{n}^{0}} \sum_{i=1}^{3}\left|U_{1 i}\right|^{2} e^{-i \frac{m_{i}^{2}-p_{n}^{2}}{2 p_{n}^{0}} T} j_{\rho}^{(2)} \bar{u}(k) \gamma^{\rho}\left(1-\gamma^{5}\right) \\
& \times \hat{p}_{n} \gamma^{\mu}\left(1-\gamma^{5}\right) v(q) j_{\mu}^{(1)} .
\end{aligned}
$$

Here $j_{\mu}^{(1)}$ and $j_{\rho}^{(2)}$ stand for the matrix elements of the charged weak hadron current associated with nuclei $1,1^{\prime}$ and $2,2^{\prime} ; k, p_{n}$, and $q$ are the 4-momenta of the electron, the intermediate virtual neutrinos and the positron, respectively, and we do not write out explicitly the fermion polarization indices.

Averaging with respect to the polarizations of the incoming nuclei and summing over the polarizations of the outgoing particles and nuclei one gets the expression for the squared amplitude as follows: 


$$
\begin{aligned}
\left\langle|M|^{2}\right\rangle= & \frac{4 G_{F}^{4}}{\left(p_{n}^{0}\right)^{2}} W_{\mu \nu}^{(1)} A^{\mu \nu \rho \sigma} W_{\rho \sigma}^{(2)} \\
& \times\left[1-4 \sum_{\substack{i, k=1 \\
i<k}}^{3}\left|U_{1 i}\right|^{2}\left|U_{1 k}\right|^{2} \sin ^{2}\left(\frac{m_{i}^{2}-m_{k}^{2}}{4 p_{n}^{0}} T\right)\right],
\end{aligned}
$$

where

$$
\begin{aligned}
A^{\mu \nu \rho \sigma}= & \frac{1}{64} \operatorname{tr}\left(\hat{p}_{n} \gamma^{\mu}\left(1-\gamma^{5}\right)(\hat{q}-m) \gamma^{\nu}\left(1-\gamma^{5}\right)\right. \\
& \left.\times \hat{p}_{n} \gamma^{\sigma}\left(1-\gamma^{5}\right)(\hat{k}+m) \gamma^{\rho}\left(1-\gamma^{5}\right)\right)
\end{aligned}
$$

(the factor $1 / 64$ is introduced in order to separate the numerical coefficient from the Lorentz structure proper), the tensors $W_{\mu \nu}^{(1)}, W_{\rho \sigma}^{(2)}$ characterizing the interaction of nuclei 1 and 2 with virtual $W$ bosons are defined as

$$
W_{\mu \nu}^{(1)}=\left\langle j_{\mu}^{(1)}\left(j_{\nu}^{(1)}\right)^{+}\right\rangle, \quad W_{\rho \sigma}^{(2)}=\left\langle j_{\rho}^{(2)}\left(j_{\sigma}^{(2)}\right)^{+}\right\rangle .
$$

Here and below, the angle brackets denote the averaging with respect to the polarizations of the incoming particles and the summation over the polarizations of the outgoing particles; i.e., in the previous formula, they denote the averaging with respect to the polarizations of nuclei 1,2 and the summation over the polarizations of nuclei $1^{\prime}, 2^{\prime}$.

Since we have dropped the neutrino masses in the time-dependent propagators, we have actually calculated the amplitude in the approximation of zero neutrino masses. As we have already noted, for macroscopic time intervals $T$ the virtual neutrinos are almost on the mass shell and, therefore, the squared momentum of the virtual neutrinos $p_{n}^{2}$ is also of the order of the neutrino masses squared and can be neglected. In other words, we may calculate the squared amplitude in the approximation $p_{n}^{2}=0$. Direct calculations show that, in this approximation, the tensor $A^{\mu \nu \rho \sigma}$ factorizes:

$$
\begin{aligned}
A^{\mu \nu \rho \sigma}= & \left(-g^{\mu \nu}\left(p_{n} q\right)+\left(p_{n}^{\mu} q^{\nu}+q^{\mu} p_{n}^{\nu}\right)+i \varepsilon^{\mu \nu \alpha \beta} p_{n \alpha} q_{\beta}\right) \\
& \times\left(-g^{\rho \sigma}\left(p_{n} k\right)+\left(p_{n}^{\rho} k^{\sigma}+k^{\rho} p_{n}^{\sigma}\right)-i \varepsilon^{\rho \sigma \alpha \beta} p_{n \alpha} k_{\beta}\right) .
\end{aligned}
$$

Correspondingly, the squared amplitude in formula (10) factorizes as follows:

$$
\begin{aligned}
\left\langle|M|^{2}\right\rangle= & \left\langle\left|M_{1}\right|^{2}\right\rangle\left\langle\left|M_{2}\right|^{2}\right\rangle \frac{1}{4\left(p_{n}^{0}\right)^{2}} \\
& \times\left[1-4 \sum_{\substack{i, k=1 \\
i<k}}^{3}\left|U_{1 i}\right|^{2}\left|U_{1 k}\right|^{2} \sin ^{2}\left(\frac{m_{i}^{2}-m_{k}^{2}}{4 p_{n}^{0}} T\right)\right],
\end{aligned}
$$

$$
\begin{aligned}
\left\langle\left|M_{1}\right|^{2}\right\rangle= & 4 G_{F}^{2}\left(-g^{\mu \nu}\left(p_{n} q\right)+\left(p_{n}^{\mu} q^{\nu}+q^{\mu} p_{n}^{\nu}\right)\right. \\
& \left.+i \varepsilon^{\mu \nu \alpha \beta} p_{n \alpha} q_{\beta}\right) W_{\mu \nu}^{(1)}, \\
\left\langle\left|M_{2}\right|^{2}\right\rangle= & 4 G_{F}^{2}\left(-g^{\rho \sigma}\left(p_{n} k\right)+\left(p_{n}^{\rho} k^{\sigma}+k^{\rho} p_{n}^{\sigma}\right)\right. \\
& \left.-i \varepsilon^{\rho \sigma \alpha \beta} p_{n \alpha} k_{\beta}\right) W_{\rho \sigma}^{(2)} .
\end{aligned}
$$

Here $\left\langle\left|M_{1}\right|^{2}\right\rangle$ is the squared amplitude of the decay process of nucleus 1 into nucleus $1^{\prime}$, positron and a massless fermion and $\left\langle\left|M_{2}\right|^{2}\right\rangle$ is the squared amplitude of the process of electron production in the collision of the massless fermion and nucleus 2 .

Now we are in a position to calculate the probability of the process depicted in diagram (2), when the time difference between the points $x$ and $y$ is equal to $T$. We will do these calculations in accordance with the rules of the standard perturbative S-matrix theory, although we are aware that the rules of calculating the probabilities of processes passing at finite time interval and finite distances may be different from those of the standard S-matrix theory. We will discuss this difference below. To this end we denote the 4-momenta of the nuclei by $P^{(i)}=\left(E^{(i)}, \vec{P}^{(i)}\right), P^{\left(i^{\prime}\right)}=\left(E^{\left(i^{\prime}\right)}, \vec{P}^{\left(i^{\prime}\right)}\right), i=1,2$, and recall that the amplitude in the momentum representation corresponding to diagram (2) contains, along with the expression in formula (9), the delta function of energymomentum conservation. Thus, to calculate the probability of the process per unit time per unit volume, we have to multiply amplitude (13) by $(2 \pi)^{4} \delta\left(P^{(1)}+P^{(2)}-P^{\left(1^{\prime}\right)}-\right.$ $\left.P^{\left(2^{\prime}\right)}-q-k\right)$ and to integrate it with respect to the momenta of the outgoing particles and nuclei.

Since the momentum $p_{n}$ of the virtual neutrinos is defined by the energy-momentum conservation in the production vertex, $p_{n}=P^{(1)}-P^{\left(1^{\prime}\right)}-q$, this integration can lead to variation in the virtual neutrino momentum, which contradicts the experimental situation in neutrino oscillations, where the virtual neutrinos propagate in the direction defined by the relative position of a source and a detector. This means that we have to calculate the differential probability of the process with $p_{n}$ fixed by the experimental setting.

Let us denote by $\vec{p}$ the momentum that is directed from the source to the detector and satisfies the momentum conservation condition $\vec{P}^{(1)}-\vec{P}^{\left(1^{\prime}\right)}-\vec{q}-\vec{p}=0$ in the production vertex and define the four-momentum $p=\left(p^{0}, \vec{p}\right)$, $p^{0}>0, p^{2}=0$. The required differential probability of the process with $p_{n}$ fixed can be obtained by multiplying amplitude (13) by the delta function $\delta\left(p_{n}-p\right)$ or, equivalently, by replacing $p_{n}$ by $p$ in the amplitude and multiplying it by $\delta\left(P^{(1)}-P^{\left(1^{\prime}\right)}-q-p\right)$. This is consistent, because we work in the approximation of massless neutrinos.

Thus, the differential probability takes the following form: 


$$
\begin{aligned}
\frac{d W}{d \vec{p}}= & \frac{G_{F}^{4}}{16(2 \pi)^{8} E^{(1)} E^{(2)}\left(p^{0}\right)^{2}}\left[1-4 \sum_{\substack{i, k=1 \\
i<k}}^{3}\left|U_{1 i}\right|^{2}\left|U_{1 k}\right|^{2} \sin ^{2}\left(\frac{m_{i}^{2}-m_{k}^{2}}{4 p^{0}} T\right)\right] \\
& \times \int d^{3} q d^{3} P^{\left(1^{\prime}\right)} d^{3} k d^{3} P^{\left(2^{\prime}\right)} \frac{1}{q^{0} E^{\left(1^{\prime}\right)} k^{0} E^{\left(2^{\prime}\right)}} \\
& \times\left(g^{\mu \nu}(p q)-\left(p^{\mu} q^{\nu}+q^{\mu} p^{\nu}\right)-i \varepsilon^{\mu \nu \alpha \beta} p_{\alpha} q_{\beta}\right) W_{\mu \nu}^{(1)}\left(P^{(1)}, P^{\left(1^{\prime}\right)}\right) \\
& \times\left(g^{\rho \sigma}(p k)-\left(p^{\rho} k^{\sigma}+k^{\rho} p^{\sigma}\right)+i \varepsilon^{\rho \sigma \alpha \beta} p_{\alpha} k_{\beta}\right) W_{\rho \sigma}^{(2)}\left(P^{(2)}, P^{\left(2^{\prime}\right)}\right) \\
& \times \delta\left(P^{(1)}+P^{(2)}-P^{\left(1^{\prime}\right)}-P^{\left(2^{\prime}\right)}-q-k\right) \delta\left(P^{(1)}-P^{\left(1^{\prime}\right)}-q-p\right) .
\end{aligned}
$$

It is easy to verify that, due to the factorization of the squared amplitude, this differential probability also factorizes. Now, since the momentum of virtual neutrinos is fixed, we can replace $T$ by $L p^{0} /|\vec{p}|$, as it was explained after formula (7), which gives

$$
\frac{d W}{d \vec{p}}=\frac{1}{2 \pi} \frac{d W_{1}}{d \vec{p}} W_{2}\left[1-4 \sum_{\substack{i, k=1 \\ i<k}}^{3}\left|U_{1 i}\right|^{2}\left|U_{1 k}\right|^{2} \sin ^{2}\left(\frac{m_{i}^{2}-m_{k}^{2}}{4|\vec{p}|} L\right)\right],
$$

where

$$
\begin{aligned}
\frac{d W_{1}}{d \vec{p}}= & \frac{1}{2 E^{(1)}} \frac{1}{(2 \pi)^{3}} \frac{1}{2 p^{0}} \int \frac{d^{3} q}{(2 \pi)^{3}} \frac{1}{2 q^{0}} \frac{d^{3} P^{\left(1^{\prime}\right)}}{(2 \pi)^{3}} \frac{1}{2 E^{\left(1^{\prime}\right)}}\left\langle\left|M_{1}\right|^{2}\right\rangle(2 \pi)^{4} \delta\left(P^{(1)}-P^{\left(1^{\prime}\right)}-q-p\right) \\
= & \frac{G_{F}^{2}}{4(2 \pi)^{5} E^{(1)} p^{0}} \int d^{3} q d^{3} P^{\left(1^{\prime}\right)} \frac{1}{q^{0} E^{\left(1^{\prime}\right)}}\left(-g^{\mu \nu}(p q)+\left(p^{\mu} q^{\nu}+q^{\mu} p^{\nu}\right)+i \varepsilon^{\mu \nu \alpha \beta} p_{\alpha} q_{\beta}\right) \\
& \times W_{\mu \nu}^{(1)}\left(P^{(1)}, P^{\left(1^{\prime}\right)}\right) \delta\left(P^{(1)}-P^{\left(1^{\prime}\right)}-q-p\right)
\end{aligned}
$$

is the differential probability of the decay of nucleus 1 into nucleus $1^{\prime}$, positron and a massless fermion with momentum $\vec{p}$, which coincides with the sum of the differential probabilities of the decay of nucleus 1 into nucleus $1^{\prime}$, positron and all the three neutrino mass eigenstates, and

$$
\begin{aligned}
W_{2}= & \frac{1}{2 E^{(2)} 2 p^{0}} \int \frac{d^{3} k}{(2 \pi)^{3}} \frac{1}{2 k^{0}} \frac{d^{3} P^{\left(2^{\prime}\right)}}{(2 \pi)^{3}} \frac{1}{2 E^{\left(2^{\prime}\right)}}\left\langle\left|M_{2}\right|^{2}\right\rangle(2 \pi)^{4} \delta\left(P^{(2)}+p-P^{\left(2^{\prime}\right)}-k\right) \\
= & \frac{G_{F}^{2}}{4(2 \pi)^{2} E^{(2)} p^{0}} \int d^{3} k d^{3} P^{\left(2^{\prime}\right)} \frac{1}{k^{0} E^{\left(2^{\prime}\right)}}\left(-g^{\rho \sigma}(p k)+\left(p^{\rho} k^{\sigma}+k^{\rho} p^{\sigma}\right)-i \varepsilon^{\rho \sigma \alpha \beta} p_{\alpha} k_{\beta}\right) \\
& \times W_{\rho \sigma}^{(2)}\left(P^{(2)}, P^{\left(2^{\prime}\right)}\right) \delta\left(P^{(2)}+p-P^{\left(2^{\prime}\right)}-k\right)
\end{aligned}
$$

is the probability of the scattering process of a massless fermion with momentum $\vec{p}$ and nucleus 2 resulting in the production of nucleus $2^{\prime}$ and an electron, which coincides with the sum of the probabilities of the scattering processes of all the three neutrino mass eigenstates and nucleus 2 . The terms in the square brackets in formula (17) reproduce the standard expression for the oscillating neutrino or electron survival probability.

The physical considerations suggest that the differential probability $\frac{d W}{d \vec{p}}$ for $L \rightarrow 0$ should be equal to the product $\frac{d W_{1}}{d \vec{p}} W_{2}$; i.e., there is an extra factor $2 \pi$ in the denominator of formula (17). This means that the standard rules of calculating the process probabilities in perturbative S-matrix theory should be modified so as to include the extra factor $2 \pi$.
The appearance of this extra factor can be explained as follows: in fact, the detector registers not only the neutrinos with momentum $\vec{p}$ from a pointlike source, but also the neutrinos with the momenta, which lie inside a small cone (see Fig. 1) with the axis along the vector $\vec{p}$. This is due to a nonzero size of the detector. Obviously, the picture has an approximate circular symmetry about the direction of momentum $\vec{p}$, which gives the factor $2 \pi$ after

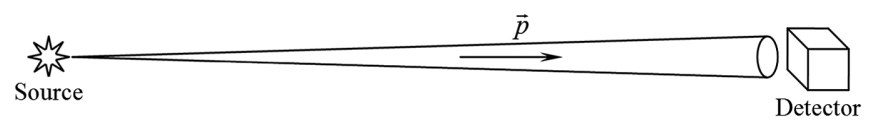

FIG. 1. Illustration of variance in the direction of the virtual neutrino momenta due to a nonzero detector size. 
the integration with respect to the azimuthal angle. Thus, the rules of the standard perturbative S-matrix theory should be modified in our case so as to include the factor $2 \pi$ along with the delta function $\delta\left(P^{(1)}-P^{\left(1^{\prime}\right)}-q-p\right)$, which fixes the 4-momentum $p$ of the intermediate neutrinos. Correspondingly, the final formula for the differential probability of the process under consideration looks like

$$
\begin{aligned}
\frac{d W}{d \vec{p}}= & \frac{1}{2 E^{(1)} 2 E^{(2)}} \int \frac{d^{3} k}{(2 \pi)^{3}} \frac{1}{2 k^{0}} \frac{d^{3} q}{(2 \pi)^{3}} \frac{1}{2 q^{0}} \frac{d^{3} P^{\left(1^{\prime}\right)}}{(2 \pi)^{3}} \frac{1}{2 E^{\left(1^{\prime}\right)}} \frac{d^{3} P^{\left(2^{\prime}\right)}}{(2 \pi)^{3}} \frac{1}{2 E^{\left(2^{\prime}\right)}}\left\langle|M|^{2}\right\rangle \\
& \times(2 \pi)^{4} \delta\left(P^{(1)}+P^{(2)}-P^{\left(1^{\prime}\right)}-P^{\left(2^{\prime}\right)}-q-k\right) 2 \pi \delta\left(P^{(1)}-P^{\left(1^{\prime}\right)}-q-p\right),
\end{aligned}
$$

which eliminates the contradiction and provides the consistent result: $\left.\frac{d W}{d \vec{p}}\right|_{L=0}=\frac{d W_{1}}{d \vec{p}} W_{2}$.

In the approximation of massless neutrinos $\frac{d W_{1}}{d \vec{p}}$ coincides with the neutrino probability flux and $W_{2}$ coincides with the cross section of the scattering process of a massless fermion on nucleus 2 . Thus, we have obtained that the probability of detecting an electron is equal to the probability of the neutrino production in the source multiplied by the probability of the neutrino interaction in the detector and the standard distance-dependent electron or neutrino survival probability; i.e., we have actually exactly reproduced the result of the standard approach to neutrino oscillations in the framework of QFT without making use of the neutrino flavor states.

In formula (17) and in the subsequent formulas, the distance $L$ appears as the distance between two neutrino interactions, which take place in the neutrino source and in the detector. However, if we consider the same process passing at a different point of the detector at a distance $L^{\prime}=L+\Delta L,|\Delta L| \ll L$, we get the same differential probability up to terms of the order $\Delta L / L$, which can be dropped. If the sizes of the source and the detector are much less than the distance between them, which is always the case in the neutrino oscillation experiments, the differential probability of all the neutrino production and detection processes passing within their volumes is the same with a very high accuracy. Therefore, the distance $L$, just like in the standard approach, can be considered as the distance between the source and the detector, and differential probability (17) can be considered as the differential probability per unit source volume and per unit detector volume. If nuclei 1 in the source have a momentum distribution, the total neutrino probability flux can be obtained by performing the average of $\frac{d W_{1}}{d \vec{p}}$ over the momentum distribution of nucleus 1 , and the number of events in the detector per unit time can be found by integrating the corresponding differential probability $\frac{d W}{d \vec{p}}$ and the densities of nuclei 1 and nuclei 2 over the volumes of the neutrino source and detector.

The rules for calculating the probabilities of neutrino oscillation processes passing at finite distances and finite time intervals were suggested by the factorized structure of their squared amplitudes arising due to the extremely small neutrino masses. However, we believe that these rules can be used for calculating the probabilities of any processes passing at finite distances and finite time intervals. In particular, they can also be used for calculating the probabilities of oscillation processes with neutral kaons, where the differential probability of the processes factorizes exactly like in formula (17) due to a simpler structure of the amplitude of such processes in the case of scalar particles.

\section{OSCILLATIONS IN EXPERIMENTS WITH NEUTRINO DETECTION BY NEUTRAL- CURRENT AND CHARGED-CURRENT INTERACTIONS}

Now we consider the case, where the neutrinos are produced in the charged-current interaction with nuclei and detected in both neutral-current and charged-current interactions with electrons, as it is done in the Kamiokande experiment. The corresponding processes are described by the following Feynman diagrams:
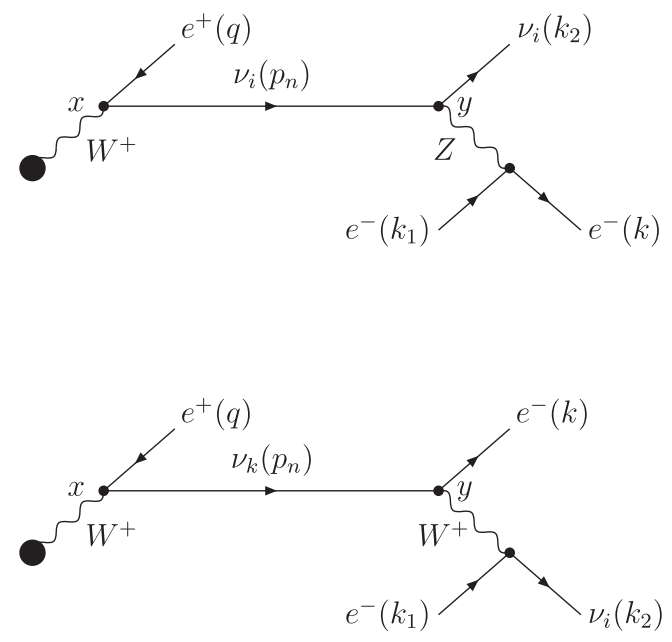

It is clear that in calculating the amplitude of the process the contribution of diagram (22) should be taken with all three neutrino mass eigenstates, i.e., $k=1,2$, 3. Since only the final electron is registered experimentally, the probabilities of the processes with different final neutrino states should 
be summed up to give the probability of registering an electron.

Now let us denote the particle momenta as follows: the momentum of the positron is $q$, the momentum of the virtual neutrinos is $p_{n}$, the momentum of the outgoing electron is $k$, the momentum of the incoming electron is $k_{1}$ and the momentum of the outgoing neutrino is $k_{2}$. Again we use the approximation of Fermi's interaction and take time-dependent propagator (7) keeping the neutrino masses only in the exponential. Then the amplitude corresponding to diagram (21) in the momentum representation looks like

$$
\begin{aligned}
M_{n c}^{(i)}= & i \frac{G_{F}^{2}}{4 p_{n}^{0}} U_{1 i}^{*} e^{-i \frac{m_{i}^{2}-p_{n}^{2}}{2 p_{n}^{0}} T} \bar{\nu}_{i}\left(k_{2}\right) \gamma^{\mu}\left(1-\gamma^{5}\right) \hat{p}_{n} \gamma^{\rho}\left(1-\gamma^{5}\right) v(q) j_{\rho} \\
& \times\left[\left(-\frac{1}{2}+\sin ^{2} \theta_{W}\right) \bar{u}(k) \gamma_{\mu}\left(1-\gamma^{5}\right) u\left(k_{1}\right)+\sin ^{2} \theta_{W} \bar{u}(k) \gamma_{\mu}\left(1+\gamma^{5}\right) u\left(k_{1}\right)\right] .
\end{aligned}
$$

Similarly, the sum over $k$ of the amplitudes corresponding to diagram (22) can be written out to be

$$
\begin{aligned}
M_{c c}^{(i)}= & -i \frac{G_{F}^{2}}{4 p_{n}^{0}} U_{1 i}^{*}\left(\sum_{k=1}^{3}\left|U_{1 k}\right|^{2} e^{-i \frac{m_{i}^{2}-p_{n}^{2}}{2 p_{n}^{0}} T}\right) \bar{u}(k) \gamma^{\mu}\left(1-\gamma^{5}\right) \hat{p}_{n} \gamma^{\rho}\left(1-\gamma^{5}\right) v(q) j_{\rho} \\
& \times \bar{\nu}_{i}\left(k_{2}\right) \gamma_{\mu}\left(1-\gamma^{5}\right) u\left(k_{1}\right) .
\end{aligned}
$$

Next, it is convenient to use the Fierz identity, which transposes the spinors $\bar{u}(k)$ and $\bar{\nu}_{i}\left(k_{2}\right)$ in the amplitude $M_{c c}^{(i)}$ and makes this amplitude look similar to $M_{n c}^{(i)}$, and to introduce the following notations for the time-dependent factors:

$$
A_{i}=U_{1 i}^{*} e^{-i \frac{m_{i}^{2}-p_{n}^{2}}{2 p_{n}^{0}} T}, \quad B_{i}=U_{1 i}^{*}\left(\sum_{k=1}^{3}\left|U_{1 k}\right|^{2} e^{-i \frac{m_{i}^{2}-p_{n}^{2}}{2 p_{n}^{0}} T}\right) .
$$

Then the total amplitude of the process with neutrino $\nu_{i}$ in the final state, which is the sum of the amplitudes $M_{n c}^{(i)}$ and $M_{c c}^{(i)}$, can be represented as follows:

$$
\begin{aligned}
M_{\mathrm{tot}}^{(i)}= & i \frac{G_{F}^{2}}{4 p_{n}^{0}} \bar{\nu}_{i}\left(k_{2}\right) \gamma^{\mu}\left(1-\gamma^{5}\right) \hat{p}_{n} \gamma^{\rho}\left(1-\gamma^{5}\right) v(q) j_{\rho} \\
& \times\left[\left(B_{i}+A_{i}\left(-\frac{1}{2}+\sin ^{2} \theta_{W}\right)\right) \bar{u}(k) \gamma_{\mu}\left(1-\gamma^{5}\right) u\left(k_{1}\right)+A_{i} \sin ^{2} \theta_{W} \bar{u}(k) \gamma_{\mu}\left(1+\gamma^{5}\right) u\left(k_{1}\right)\right] .
\end{aligned}
$$

Now, we have to calculate the squared amplitude, averaged with respect to the polarizations of the incoming nucleus and particles and summed over the polarizations of the outgoing nucleus and particles. Similar to the case of the neutrino detection in charged-current interaction, in the approximation $p_{n}^{2}=0$ the squared amplitude factorizes as follows:

$$
\begin{gathered}
\left\langle\left|M_{\mathrm{tot}}^{(i)}\right|^{2}\right\rangle=\left\langle\left|M_{1}\right|^{2}\right\rangle\left\langle\left|M_{2}^{(i)}\right|^{2}\right\rangle \frac{1}{4\left(p_{n}^{0}\right)^{2}}, \\
\left\langle\left|M_{1}\right|^{2}\right\rangle=4 G_{F}^{2}\left(-g^{\mu \nu}\left(p_{n} q\right)+\left(p_{n}^{\mu} q^{\nu}+q^{\mu} p_{n}^{\nu}\right)+i \varepsilon^{\mu \nu \alpha \beta} p_{n \alpha} q_{\beta}\right) W_{\mu \nu}^{(1)}, \\
\left\langle\left|M_{2}^{(i)}\right|^{2}\right\rangle=64 G_{F}^{2}\left[\left|B_{i}+A_{i}\left(-\frac{1}{2}+\sin ^{2} \theta_{W}\right)\right|^{2}\left(p_{n} k_{1}\right)^{2}+\left|A_{i}\right|^{2} \sin ^{4} \theta_{W}\left(p_{n} k\right)^{2}\right. \\
\left.-\sin ^{2} \theta_{W} \operatorname{Re}\left(\left(B_{i}+A_{i}\left(-\frac{1}{2}+\sin ^{2} \theta_{W}\right)\right) A_{i}^{*}\right)\left(p_{n} k_{2}\right) m^{2}\right]
\end{gathered}
$$

Here, $\left\langle\left|M_{1}\right|^{2}\right\rangle$ is the squared amplitude of the decay process of nucleus 1 into nucleus $1^{\prime}$, positron and a massless fermion, $W_{\mu \nu}^{(1)}$ denoting the corresponding averaged product of the matrix elements of the charged weak hadron current, and $\left\langle\left|M_{2}^{(i)}\right|^{2}\right\rangle$ is the squared amplitude of the scattering process of a massless fermion and the incoming electron. 
As we have found in the previous section for the case of neutrino registration in charged-current interaction, to obtain the differential probability of the process, we have to multiply the amplitude $\left\langle\left|M_{\mathrm{tot}}^{(i)}\right|^{2}\right\rangle$ by the delta function of energy-momentum conservation $(2 \pi)^{4} \delta\left(P^{(1)}+k_{1}-\right.$ $\left.P^{\left(1^{\prime}\right)}-q-k-k_{2}\right)$ and by the delta function $2 \pi \delta\left(P^{(1)}-\right.$ $\left.P^{\left(1^{\prime}\right)}-q-p\right)$ that selects the momentum of the virtual neutrinos, substitute $p$ instead of $p_{n}$ in it and to integrate with respect to the momenta of the outgoing particles and nucleus. This gives

$$
\begin{gathered}
\frac{d W^{(i)}}{d \vec{p}}=\frac{d W_{1}}{d \vec{p}} W_{2}^{(i)}, \\
\frac{d W_{1}}{d \vec{p}}=\int \frac{\left\langle\left|M_{1}\right|^{2}\right\rangle}{2 E^{(1)}}(2 \pi)^{4} \delta\left(P^{(1)}-P^{\left(1^{\prime}\right)}-q-p\right) \\
\times \frac{d^{3} q}{(2 \pi)^{3} 2 q^{0}} \frac{d^{3} P^{\left(1^{\prime}\right)}}{(2 \pi)^{3} 2 E^{\left(1^{\prime}\right)}} \frac{1}{(2 \pi)^{3} 2 p^{0}}, \\
W_{2}^{(i)}=\int \frac{\left\langle\left|M_{2}^{(i)}\right|^{2}\right\rangle}{2 p^{0} 2 k_{1}^{0}}(2 \pi)^{4} \delta\left(k_{1}+p-k-k_{2}\right) \\
\times \frac{d^{3} k}{(2 \pi)^{3} 2 k^{0}} \frac{d^{3} k_{2}}{(2 \pi)^{3} 2 k_{2}^{0}} .
\end{gathered}
$$

Formula (31) means that $\frac{d W_{1}}{d \vec{p}}$ is the differential decay probability of nucleus 1 into nucleus $1^{\prime}$, positron and a massless neutral fermion with fixed momentum $\vec{p}$. In fact, this is the sum of the differential decay probabilities of nucleus 1 into nucleus $1^{\prime}$, positron and all the three neutrino mass eigenstates $\nu_{i}$ with fixed momentum $\vec{p}$ taken in the approximation of zero neutrino masses. Similarly, in accordance with formula (32), the probability $W_{2}^{(i)}$ is the probability of the process of scattering of a massless neutral fermion and electron with the production of electron and the neutrino mass eigenstate $\nu_{i}$ in the final state. Obviously, this probability is the sum of the probabilities of the processes of scattering of electron and all the three neutrino mass eigenstates with the production of electron and the neutrino mass eigenstate $\nu_{i}$ in the final state.

To obtain the probability of finding an electron in the final state we have to sum the probability $\frac{d W^{(i)}}{d \vec{p}}$ over $i=1,2,3$. Obviously, this reduces to summing over $i$ the squared amplitude $\left\langle\left|M_{2}^{(i)}\right|^{2}\right\rangle$, because only this amplitude depends on $i$.

Since now the virtual neutrinos have fixed momentum $p$, we can replace $T$ by $L p^{0} /|\vec{p}|$ in all the subsequent formulas. Then the definition of the time-dependent factors $A_{i}$ and $B_{i}$ in (25) leads to the following expressions for their absolute values and products:

$$
\begin{gathered}
\left|A_{i}\right|^{2}=\left|U_{1 i}\right|^{2}, \\
\left|B_{i}\right|^{2}=\left|U_{1 i}\right|^{2}\left[1-4 \sum_{\substack{k, l=1 \\
k<l}}^{3}\left|U_{1 k}\right|^{2}\left|U_{1 l}\right|^{2} \sin ^{2}\left(\frac{m_{k}^{2}-m_{l}^{2}}{4|\vec{p}|} L\right)\right],
\end{gathered}
$$

$$
\operatorname{Re}\left(A_{i} B_{i}^{*}\right)=\left|U_{1 i}\right|^{2} \sum_{k=1}^{3}\left|U_{1 k}\right|^{2} \cos ^{2}\left(\frac{m_{k}^{2}-m_{i}^{2}}{4|\vec{p}|} L\right) .
$$

Substituting these expressions into formula (29) and summing over $i$, we get

$$
\begin{aligned}
\sum_{i=1}^{3}\left\langle\left|M_{2}^{(i)}\right|^{2}\right\rangle= & 64 G_{F}^{2}\left[\sin ^{4} \theta_{W}(p k)^{2}+\left(\left(\frac{1}{2}+\sin ^{2} \theta_{W}\right)^{2}-8 \sin ^{2} \theta_{W} \sum_{\substack{k, l=1 \\
k<l}}^{3}\left|U_{1 k}\right|^{2}\left|U_{1 l}\right|^{2} \sin ^{2}\left(\frac{m_{k}^{2}-m_{l}^{2}}{4|\vec{p}|} L\right)\right)\left(p k_{1}\right)^{2}\right. \\
& \left.-\left(\sin ^{2} \theta_{W}\left(\frac{1}{2}+\sin ^{2} \theta_{W}\right)-4 \sin ^{2} \theta_{W} \sum_{\substack{k, l=1 \\
k<l}}^{3}\left|U_{1 k}\right|^{2}\left|U_{1 l}\right|^{2} \sin ^{2}\left(\frac{m_{k}^{2}-m_{l}^{2}}{4|\vec{p}|} L\right)\right)\left(p k_{2}\right) m^{2}\right] .
\end{aligned}
$$

Next, substituting this expression into formula (32) summed over $i$ and evaluating the integrals with the help of the formulas for neutrino-electron scattering kinematics presented in chapter 16 of Ref. [13], we arrive at the following result:

$$
\begin{aligned}
W_{2}= & \frac{G_{F}^{2} m}{2 \pi} \frac{2|\vec{p}|^{2}}{2|\vec{p}|+m}\left[1-2 \sin ^{2} \theta_{W}\left(1+\frac{2|\vec{p}|}{2|\vec{p}|+m}\right)+4 \sin ^{4} \theta_{W}\left(1+\frac{1}{3}\left(\frac{2|\vec{p}|}{2|\vec{p}|+m}\right)^{2}\right)\right. \\
& \left.+4 \sin ^{2} \theta_{W}\left(1+\frac{2|\vec{p}|}{2|\vec{p}|+m}\right)\left(1-4 \sum_{\substack{k, l=1 \\
k<l}}^{3}\left|U_{1 k}\right|^{2}\left|U_{1 l}\right|^{2} \sin ^{2}\left(\frac{m_{k}^{2}-m_{l}^{2}}{4|\vec{p}|} L\right)\right)\right] .
\end{aligned}
$$

We see that, in the case where the neutrinos are produced in the charged-current interaction with nuclei and detected in both neutral-current and charged-current interactions with electrons, the situation is different from the case, where neutrinos are detected in charged-current interactions. The same situation takes place in the standard approach, and the formula for $W_{2}$ exactly coincides with the expression $P_{\nu_{e} \rightarrow \nu_{e}} \sigma_{\nu_{e} e}+\left(1-P_{\nu_{e} \rightarrow \nu_{e}}\right) \sigma_{\nu_{\mu} e}$, which one expects for this quantity in the standard approach. 


\section{CONCLUSION}

In the present paper, we have shown that it is possible to calculate consistently neutrino oscillation processes in a quantum field-theoretical approach within the framework of the SM minimally extended by the right neutrino singlets using only plane wave states of the particles. To this end we have adapted the standard formalism of perturbative S-matrix for calculating the amplitudes of the processes passing at finite distances and finite time intervals by modifying the Feynman propagator in the momentum representation. The developed approach is physically transparent and, unlike the quantum mechanical description in terms of plane waves based on the notion of neutrino flavor states, has the advantage of not violating energy-momentum conservation. It can be considered as a formalization of the approach based on the use of the Feynman propagators and wave packets [4-7], which allows one to simplify the calculations essentially. In its framework, the calculation of amplitudes is carried out in the way that is most similar to the one used in the usual perturbative S-matrix formalism.

The application of this modified formalism to describing the neutrino oscillation processes with neutrino detection by charged-current and neutral-current interactions with electrons showed that the standard results can be easily and consistently obtained using only the mass eigenstates of these particles. There is a good reason to believe that the developed approach is equivalent to the approach based on the use of the Feynman propagators and wave packets just like the Feynman diagram technique using plane wave states of particles is equivalent to the calculations of the amplitudes of scattering processes with the help of wave packets. However, the use of plane waves, which essentially simplifies the calculation of amplitudes, results in the formalism in its present form being unable to reproduce the coherence and localization terms, which naturally arise in the approaches using wave packets. Thus, the problem of describing the coherence and localization properties of neutrino oscillations in this approach needs a special investigation.

\section{ACKNOWLEDGMENTS}

The authors are grateful to E. Boos, A. Lobanov, and M. Smolyakov for reading the manuscript and making important comments and to L. Slad for useful discussions. Analytical calculations of the amplitudes have been carried out with the help of the COMPHEP and REDUCE packages. The work was supported by Grant No. NSh7989.2016.2 of the President of the Russian Federation.
[1] C. Giunti and C. W. Kim, Fundamentals of Neutrino Physics and Astrophysics (Oxford University Press, New York, 2007).

[2] S. Bilenky, Introduction to the physics of massive and mixed neutrinos, Lect. Notes Phys. 817, 1 (2010).

[3] K. Nakamura and S. T. Petcov (Particle Data Group), Neutrino masses, mixing, and oscillations, Chin. Phys. C 38, 090001 (2014).

[4] C. Giunti, C. W. Kim, J. A. Lee, and U. W. Lee, On the treatment of neutrino oscillations without resort to weak eigenstates, Phys. Rev. D 48, 4310 (1993).

[5] W. Grimus and P. Stockinger, Real oscillations of virtual neutrinos, Phys. Rev. D 54, 3414 (1996).

[6] M. Beuthe, Oscillations of neutrinos and mesons in quantum field theory, Phys. Rep. 375, 105 (2003).
[7] E. K. Akhmedov and J. Kopp, Neutrino oscillations: Quantum mechanics vs. quantum field theory, J. High Energy Phys. 04 (2010) 008; 10 (2013) 52.

[8] A. G. Cohen, S. L. Glashow, and Z. Ligeti, Disentangling Neutrino Oscillations, Phys. Lett. B 678, 191 (2009).

[9] A. E. Lobanov, Oscillations of particles in the Standard Model, Teor. Mat. Fiz. 192, 70 (2017); Theor. Math. Phys. 1921000 (2017).

[10] I. P. Volobuev, Quantum field-theoretical description of neutrino and neutral kaon oscillations, arXiv:1703.08070.

[11] R. P. Feynman, Space-time approach to quantum electrodynamics, Phys. Rev. 76, 769 (1949).

[12] N. N. Bogoliubov and D. V. Shirkov, Introduction to the Theory of Quantized Fields, 3rd edition (Wiley, New York, 1980).

[13] L. B. Okun, Leptons and Quarks (North Holland, New York, 1984). 